\title{
Clinical Significance of Blood Pressure Levels during Treadmill Exercise Testing
}

\author{
${ }^{1}$ Sandeep Bansal, ${ }^{2}$ Anwar H Ansari
}

\begin{abstract}
Treadmill exercise testing is the most common noninvasive test to assess patients with suspected or known cardiovascular disease. Measurement of blood pressure (BP) during exercise stress is cumbersome due to patient motion and hemodynamic changes during exercise. Different population studies give differing levels for abnormal BP response to exercise and there is no unanimity about the level of BP at which exercise test is contraindicated or terminated. However, studies uniformly associate abnormal BP responses to exercise not only with risk of future hypertension but also with cardiovascular events, stroke and mortality. The present article provides a brief account of common precautions in BP measurement, the diagnostic and prognostic significance of different BP responses during exercise stress testing.
\end{abstract}

Keywords: Exercise stress test, Hypertensive response, Hypotensive response, Recovery phase.

How to cite this article: Bansal S, Ansari AH. Clinical Significance of Blood Pressure Levels during Treadmill Exercise Testing. Hypertens J 2015;1(2):83-87.

\section{Source of support: Nil}

Conflict of interest: None

\section{INTRODUCTION}

Exercise stress testing is the most frequent noninvasive test to assess patients with suspected or proven cardiovascular disease. The test is used to determine functional capacity, extent and likelihood of coronary artery disease (CAD), effects of therapy and estimate prognosis. Exercise is a common physiological stress used to assess cardiovascular abnormalities that are not present at rest. During exercise testing, apart from electrocardiogram (ECG) changes, patient's symptoms, abnormal hemodynamics, heart rate response and functional capacity are important determinants used in assessing long-term prognosis.

\footnotetext{
${ }^{1}$ Consultant, Professor and Head, ${ }^{2}$ Assistant Professor

1Department of Cardiology, Safdarjung Hospital, New Delhi, India

${ }^{2}$ Department of Cardiology, Vardhman Mahavir Medical College and Safdarjung Hospital, New Delhi, India

Corresponding Author: Sandeep Bansal, Consultant Professor and Head, Department of Cardiology, Safdarjung Hospital, New Delhi, India, Phone: 9810543368, e-mail: drsbansal2000@yahoo.com
}

The manual determination of blood pressure (BP) is still widely used routinely for measurement of BP. It can be difficult to define the figures for systolic blood pressure (SBP) and diastolic blood pressure (DBP) during exercise testing, especially the latter because DBP during effort may be heard till $0 \mathrm{~mm} \mathrm{Hg}$ if fifth Korotkov sound is used to measure DBP, so the fourth sound must be used to define DBP. The determination of BP through automated equipment with right technique offers many advantages over manual technique and should be used wherever possible.

The abnormal BP response can develop during the test, in the recovery phase or even prior to exercise. It is important to take into account the safety BP levels during exercise testing, both of contraindications and the test termination levels. The relevance of BP response to exercise lies not only for future hypertension but also for cardiovascular events, stroke and mortality.

\section{EXERCISE AND BLOOD PRESSURE}

Blood pressure response to exercise depends on age, sex, ethnicity, level of physical fitness and cardiovascular status. Normally, SBP increases progressively with exercise with increasing workloads to a peak of 160 to $200 \mathrm{~mm} \mathrm{Hg}$, with older individuals having higher BP due to less compliant vascular wall. ${ }^{1}$

As a group, blacks tend to develop higher SBP response than whites. In normal individuals, the DBP usually does not change significantly and may even fall as a result of decrease of peripheral vascular resistance (PVR).

\section{POSTEXERCISE PHYSIOLOGICAL RESPONSE}

An acute fall in BP minutes or hours after exercise termed postexercise hypotension ${ }^{2}$ typically constitutes $\mathrm{BP}$ decreases of 5 to $20 \mathrm{~mm} \mathrm{Hg}$ for hours after exercise. Strong relationship has been observed between acute postexercise hypotension and chronic reduction in $\mathrm{BP}$ among prehypertensive individuals suggestive that acute postexercise hypotension might well identify achievable decrease in BP by exercise training. ${ }^{3}$

\section{BLOOD PRESSURE MEASUREMENT}

Accurate BP measurement during exercise is difficult to obtain. Ellestad ${ }^{4}$ found that simple hearing differences 
and observer bias among individuals led to 'significant intra- and interobserver variability 'even when the common sources of BP measurement error (inaccurate manometers, inappropriate sized cuffs, too rapid deflation rates and training issues) were eliminated. These issues can be further confounded by the noise of the patient on treadmill.

The manual method is still recommended during exercise testing. The central BP or its equivalent, the brachial pressure is validated for a proper diagnosis and prognosis of cardiovascular morbidity and mortality. ${ }^{5}$

In general, the automatic measurements of BP correlate well with manual methods and have clinically acceptable absolute differences. The SunTech Tango ${ }^{\circledR}$ exercise BP monitor (SunTech Medical Instruments, NC,USA) is an automated auscultatory BP monitor which uses $\mathrm{R}$ wave gated brachial sphygmomanometry incorporating noise reducing signal processing specifically implemented to allow extraction and identification of Korotkov sounds, and thus SBP and DBP during exercise.

\section{ABNORMAL BP RESPONSES DURING TREADMILL TESTING}

- Hypertensive response

- Hypotensive response

- Low systolic pressure peak.

\section{Hypertensive Response}

The exercise hypertension is defined according to Framingham criteria, namely, a peak SBP during exercise greater than $210 \mathrm{~mm} \mathrm{Hg}$ in men and $190 \mathrm{~mm} \mathrm{Hg}$ in women. ${ }^{1}$ Systolic blood pressure max effort is commonly used but there are figures at different effort levels. ${ }^{6}$ Although the relevance of the diagnosis and prognosis of a HR is not fully clarified, some of these patients have an increased risk of future hypertension, ${ }^{6,7}$ left ventricular hypertrophy or abnormal mobility, ${ }^{8-10}$ stroke, $^{10,11}$ cardiovascular incidents, ${ }^{10}$ increased mortality ${ }^{12,13}$ and endothelial dysfunction. ${ }^{14}$

Other findings related to HR are hypercholesterolemia, ${ }^{15}$ increased angiotensin $\mathrm{II}^{16}{ }^{16}$ inflammatory markers, such as leukocytes, ${ }^{17}$ carotid atherosclerosis, ${ }^{18}$ arterial stiffness and albuminuria. ${ }^{19}$

\section{Mechanism Responsible for Exercise Hypertension}

Although the exact mechanism responsible for exaggerated $\mathrm{BP}$ response to exercise has not been elucidated, there are some plausible mechanisms. Wilson et $\mathrm{al}^{20}$ found that the total peripheral resistance in those with exercise hypertension did not fall adequately to compensate for the rise in cardiac output during exercise. Accordingly, the exercise hypertension can partially be explained by increased PVR and impaired capacity for exercise induced vasodilatation. These responses of peripheral vascular function can be explained by a hyper-reactivity of sympathetic nerves and an increased vascular response to adrenergic stimulation or by a thickening of the arteriolar wall that alters its ability to respond to vasoconstrictor stimuli. ${ }^{21}$ Among those patients with these characteristics, higher cardiac output not only raises the SBP but also causes marked DBP elevations like those occurring in established hypertension. Moreover, several studies have found that normotensive individuals with hypertensive response to exercise present changes in the heart structure and function that are usually observed in the early course of the hypertension disease. ${ }^{8}$

Some important implications of hypertensive response include the following:

\section{Hypertensive Response as Predictor of Future Hypertension}

Early detection of hypertension can prevent critical damage to key organs. Several studies found that an exaggerated BP response to physical effort is a prognostic factor for hypertension even in children. ${ }^{22}$ In general, this shows low sensitivity (25-40\%) with high specificity (73-90\%). ${ }^{11,23}$

Increased BP response to exercise is remarkable in patients with hypertension compared with normotensives. ${ }^{24}$ It has been suggested that this type of response to exercise can be used as an additional risk marker for hypertension.

A finding of another study was a high prevalence of masked hypertension in apparently healthy patients presenting with normal office BP during a clinically indicated exercise stress test. ${ }^{25}$ The prevalence of masked hypertension in HR to exercise patients from that study was four fold this value highlighting the importance of an exercise stress test as a screening tool for masked hypertension. $^{25}$

The finding that an individual's risk of developing hypertension in those with high normal BP was greatly increased if they exhibit an exaggerated BP response to exercise confirms an incremental contribution of exercise $\mathrm{BP}$ response above resting $\mathrm{BP}$ in predicting future hypertension. Hence, exercise testing in populations at high risk of hypertension could provide important additional information concerning hypertension risk. ${ }^{26}$

\section{Cardiovascular Events}

In Framingham Heart Study, an exaggerated BP response to submaximal exercise was associated with significant increase in incidence of cardiovascular events over a 20 years follow-up. ${ }^{27}$ 


\section{Hypotensive Response}

Although there is no consensus aggreement on its definition, it is most frequently defined as SBP during exercise falling below resting systolic pressure. Another definition is an initial rise in SBP followed by a fall of $20 \mathrm{~mm} \mathrm{Hg}$ or more. Either of these definitions would be an absolute reason to terminate the exercise test. The first has a significant relative risk of 3.2 for cardiovascular events, while the predictive value of the second is lower. The prevalence varies between 5 and $8 \%$ and was higher in patients with coronary heart disease. ${ }^{28}$ The former definition is more predictive of a poor prognosis and is usually related to severe multivessel CAD with left ventricular dysfunction, especially when noted with other signs of ischemia, such as ST depression or angina at a low workload. Its positive predictive value is high in men but much lower in women. Its presence usually warrants consideration of prompt invasive evaluation. Exercise-associated hypotension may also be seen in patients with cardiomyopathy, left ventricular outflow tract obstruction, enhanced vagal tone, hypovolemia, antihypertensive medications and arrhythmias.

This hypotensive response must be differentiated from 'pseudo-exercise-induced hypotension,' which occurs in patients who are anxious about the exercise study and begin exercise with a somewhat elevated systolic pressure. As exercise proceeds in the first stage, this elevated pressure usually settles down or 'falls' toward its resting level. As exercise continues, there is a gradual rise in BP.

\section{Low Systolic Pressure Peak}

This is defined as a rise to less than $140 \mathrm{~mm} \mathrm{Hg}$ or a lower than $10 \mathrm{~mm} \mathrm{Hg}$ rise overall. After excluding poor exercise effort, this response is often associated with severe CAD and worse cardiovascular outcomes in persons with or without known CAD and warrants further evaluation.

\section{ABNORMAL BP RESPONSE DURING RECOVERY (TABLE 1)}

Huang et $\mathrm{al}^{29}$ retrospectively studied 3054 patients referred for exercise testing with 10 years follow-up.

Table 1: Recovery and cardiac events

\begin{tabular}{|c|c|c|c|c|}
\hline $\begin{array}{l}\text { Years } \\
\text { (source) }\end{array}$ & $\begin{array}{l}\text { Population } \\
\text { (number) }\end{array}$ & $\begin{array}{l}\text { Follow-up } \\
\text { (years) }\end{array}$ & Protocol & $\begin{array}{l}\text { Definition HR } \\
(\mathrm{mm} \mathrm{Hg})\end{array}$ \\
\hline $2008^{29}$ & 3,054 & 10 & Bruce & SBPrec $3^{\prime}>1^{\prime}$ \\
\hline $2006^{30}$ & $\begin{array}{l}\text { Normotensive } \\
\text { (86) }\end{array}$ & 5 & Bruce & $\begin{array}{l}\text { BP > }>160 / 90 \\
\text { at } 5^{\prime}\end{array}$ \\
\hline $2004^{31}$ & $\begin{array}{l}\text { Normotensive } \\
(2,336)\end{array}$ & 13.1 & Cycle & $\begin{array}{l}\text { SBPrec >195 } \\
\text { at 2' }\end{array}$ \\
\hline $1999^{6}$ & $\begin{array}{l}\text { Normotensive } \\
(2,310)\end{array}$ & 8 & Bruce & $\begin{array}{l}\text { SBP } 142 \pm 19 \\
\text { at } 3 \text {, }\end{array}$ \\
\hline
\end{tabular}

A paradoxical increase in SBP after exercise (SBP to 3 minutes recovery $\geq 1$ minute of recovery) is an important and significant predictor of cardiovascular mortality with an adjusted odds ratio of 1.80 .

Yosefy et $\mathrm{al}^{30}$ studied 86 apparently healthy patients who came for a routine check up, with an average age of $60 \pm 4.1$ (46-75) years, with Bruce protocol, measuring the SBP and DBP at 5 minutes of recovery. After 5 years of follow-up, those who had a HR, defined as $>160 / 90 \mathrm{~mm}$ $\mathrm{Hg}$ developed a worse cardiovascular profile, and other adversities (abnormal cholesterol levels, hypertension and cardiovascular disease and stroke combined) with a RR of 1.32 .

Laukkanen et $\mathrm{al}^{31}$ studied 2336 individuals on cycloergometer with average age of $52.9 \pm 5.1$, with a follow-up of 13.1 years. The average $\mathrm{SBP}_{\max }$ was $202 \mathrm{~mm} \mathrm{Hg}$, and at 2 minutes was $183 \mathrm{~mm} \mathrm{Hg}$. The SBP >195 mm Hg at 2 minutes of recovery was associated with a risk of myocardial infarction of 1.7. The adjusted RR was 1.45 for cardiovascular mortality and 1.68 for myocardial infarction.

Singh et $\mathrm{al}^{6}$ studied 1026 men and 1284 women with an average age of $42 \pm 10$ years in the Framingham offspring study, normotensive, with 8 years follow-up. They found that a recovery SBP at 3 minutes was predictive of hypertension in men, with an adjusted RR of 1.92 (average recovery SBP at 3 minutes of $142 \pm 19 \mathrm{~mm} \mathrm{Hg}$ ).

\section{CONTRAINDICATIONS FOR EXERCISE TESTING}

The American College of Cardiology/American Heart Association guidelines do not refer to any absolute contraindication to a resting BP while the Spanish guidelines mention a resting $\mathrm{BP}$ of $240 / 130 \mathrm{~mm} \mathrm{Hg}$ as absolute contraindication to exercise testing. In the ACC/ AHA guidelines, a relative contraindication to exercise test is shown as severe arterial hypertension (suggested definitions: SBP > 200 and/or DBP > $110 \mathrm{~mm} \mathrm{Hg}){ }^{32}$

\section{BLOOD PRESSURE RELATED INDICATIONS FOR TERMINATING EXERCISE TESTING}

\section{Absolute}

- Drop in SBP > $10 \mathrm{~mm} \mathrm{Hg}$ from baseline BP despite an increase in workload, when accompanied by other evidence of ischemia

- Technical difficulties in monitoring ECG or SBP.

\section{Relative}

- Drop in SBP $\geq 10 \mathrm{~mm} \mathrm{Hg}$ from baseline BP despite an increase in workload, in the absence of other evidence of ischemia

- Hypertensive response (SBP > $250 \mathrm{~mm} \mathrm{Hg}$ and/or a DBP > 115 mm Hg. ${ }^{32}$ 


\section{SAFETY OF EXERCISE TESTING}

Exercise testing has excellent safety record. The risk is determined by the patient's clinical status referred for the procedure. In nonselected patient populations, the mortality is $<0.01 \%$ and morbidity is $<0.05 \%$. The risk is greater when it is performed soon after an acute ischemic event or patients with critical obstruction to left ventricular outflow tract.

With regard to the $\mathrm{BP}$, and without having defined evidence and with the aim of avoiding serious complications, it is best to follow the current recommendations of contraindication and of termination of exercise testing. One of the main reference guides is the that of the Committee of the Stress Tests of the American College of Cardiology and American Heart Association, which also follows the American College of Sports Medicine. ${ }^{32,33}$

\section{CONCLUSION}

There are many studies that have been undertaken regarding diagnostic and prognostic value of abnormal $\mathrm{BP}$ response to exercise testing during effort, recovery period or even before commencing exercise. However, these studies have used different methods for diagnosing abnormal BP response and different exercise protocols. Hence, no standard values have been determined, which could have global acceptance. Inspite of all these shortcomings, there is undoubtedly, evidence that an abnormal BP response to exercise is related to increased risk of future hypertension, cardiovascular events and mortality. There is additional incremental contribution of hypertensive $\mathrm{BP}$ response to exercise above resting $\mathrm{BP}$ in predicting subsequent hypertension in normotensive individuals. Conversely, in case of hypotensive or insufficient BP response a possible ischemic cardiomyopathy, obstructive ventricular outflow disease or left ventricular dysfunction should be suspected and ventricular function assessed. However, despite its prognostic value in predicting future hypertension, routine exercise testing in normotensive individuals to assess the risk of future hypertension cannot be recommended at present due to very low yield. More studies are needed to reach a consensus regarding abnormal response to exercise during effort.

\section{REFERENCES}

1. Lauer MS, Levy D, Anderson KM, et al. Is there a relationship between exercise systolic blood pressure response and left ventricular mass? The Framingham Heart Study. Ann Inter Med 1992 Feb 1;116(3):203-210.

2. Eicher JD, Maresh CM, Tsongalis GJ, et al. The additive blood pressure lowering effects of exercise intensity on post exercise hypotension. Am Heart J 2010 Sep;160(3):513-520.
3. Liu S, Goodman J, Nolan R, et al. Blood pressure responses to acute and chronic exercise are related in prehypertension. Med Sci Sports Exerc 2012 Sep;44(9):1644-1652.

4. Ellestad MH. Stress Testing: Principles and Practice-Oxford University Press, USA, 2003. p. 335-338.

5. O'Rourke MF, Seward JB. Central arterial pressure and arterial pressure pulse: new views entering the second century after Korotkov. Mayo Clin Proc 2006 Aug;81(8):1057-1068.

6. Singh JP, Larson MG, Manolio TA, et al. Blood pressure response during Treadmill testing as a risk factor for new-onset hypertension. The Framingham heart study. Circulation 1999 Apr;99(14):1831-1836.

7. Pescatello LS, Franklin BA, Fagard R, et al. American College of Sports Medicine. American College of Sports Medicine position stand. Exercise and hypertension. Med Sci Sports Exerc 2004 Mar;36(3):533-53. Review.

8. Gottdiener JS, Brown J, Zoltick J, et al. Left ventricular hypertrophy in men with normal blood pressure: relation to exaggerated blood pressure response to exercise. Ann Intern Med 1990 Feb;112(3):161-166.

9. Sung J, Ouyang P, Silber HA, et al. Exercise blood pressure response is related to left ventricular mass. J Hum Hypertens 2003 May;17(5):333-338.

10. Ha JW, Juracan EM, Mahoney DW, et al. Hypertensive response to exercise: a potential cause for new wall motion abnormality in the absence of coronary artery disease. J Am Coll Cardiol 2002 Jan;39(2):323-327.

11. Allison TG, Cordeiro MA, Miller TD, et al. Prognostic significance of exercise-induced systemic hypertension in healthy subjects. Am J Cardiol 1999 Feb;83(3):371-375.

12. Gupta MP, Polena S, Coplan N, et al. Prognostic significance of systolic blood pressure increases in men during exercise stress testing. Am J Cardiol 2007 Dec;100(11):1609-1613.

13. Naughton J, Dorn J, Oberman A, et al. Maximal exercise sys_ tolic pressure, exercise training, and mortality in myocardial infarction patients. Am J Cardiol 2000 Feb;85(4):416-420.

14. Chang HJ, Chung J, Choi SY, et al. Endothelial dysfunction in patients with exaggerated blood pressure response during treadmill test. Clin Cardiol 2004 July;27(7):421-425.

15. Kavey RE, Kveselis DA, Gaum WE, et al. Exaggerated blood pressure response to exercise in children with increased lowdensity lipoprotein cholesterol. Am Heart J 1997 Feb;133(2):162-168.

16. Shim CY, Ha JW, Park, et al. Exaggerated blood pressure response to exercise is associated with augmented rise of angiotensin II during exercise. J Am Coll Cardiol 2008;52(4):287-292.

17. Jae SY, Fernhall B, Lee M, et al. Exaggerated blood pressure response to exercise is associated with inflammatory markers. J Cardiopulm Rehabil 2006 May-Jan;26(3):145-149.

18. Jae SY, Fernhall B, Heffernan KS, et al. Exaggerated blood pressure response to exercise is associated with carotid atherosclerosis in apparently healthy men. J Hypertens 2006 May;24(5):881-887.

19. Tsioufis C, Dimitriadis K, Thomopoulos C, et al. Exercise blood pressure response, albuminuria, and arterial stiffness in hypertension. Am J Med 2008;121(10):894-902.

20. Wilson MF, Sung BH, Pincomb GA, et al. Exaggerated pressure response to exercise in men at risk for systemic hypertension. Am J Cardiol 1990;66(7):731-736.

21. Kavey RW, Kveselis DA, Gaum WE. Exaggerated blood pressure response to exercise in children with increased low-density lipoprotein cholesterol. Am Heart J 1997 Feb;133(2):162-168. 
22. Lauer RM, Burns TL, Clarke WR, et al. Childhood predictors of future blood pressure. Hypertension 1991 Sep;18(Suppl3): I74-I81.

23. Matthews CE, Pate RR, Jackson KL, et al. Exaggerated blood pressure response to dynamic exercise and risk of future hypertension. J Clin Epidemiol 1998 Jan;51(1):29-35.

24. deSouza NS, Gomides RS, daSilva DV, et al. Intra-arterial blood pressure response in hypertensive subjects during low and high intensity resistance exercise. Sao Paulo 2010 Mar;65(3):271-277.

25. Sharman JE, Hare JL, Thomas S, et al. Association of masked hypertension and left ventricular remodelling with hypertensive response to exercise. Am J Hypertens 2011 Aug;24(8): 898-903.

26. Miyai N, Arita M, Morioka J, et al. Exercise BP response in subjects with high normal BP: exaggerated BP response to exercise and risk of future hypertension in subjects with high normal BP. J Am Coll Cardiol 2000;36(5):1626-1631.

27. Lewis GD,Gona P, Larson MG, et al. Exercise blood pressure and the risk of incident cardiovascular disease (from the Framingham Heart Study). Am J Cardiol 2008;101(11):1614.

28. Le VV, Mitiku T, Sungar G, et al. The blood pressure response to dynamic exercise testing: a systematic review. Prog Cardiovasc Dis 2008 Sep-Oct;51(2):135-160.
29. Huang CL, Su TC, Chen WJ, et al. Usefulness of paradoxical systolic blood pressure increase after exercise as a predictor of cardiovascular mortality. Am J Cardiol 2008;102(5): 518-523.

30. Yosefy C, Jafari J, Klainman E, et al. The prognostic value of post-exercise blood pressure reduction in patients with hypertensive response during exercise stress test. Int J Cardiol 2006 Aug;111(3):352-357.

31. Laukkanen JA, Kurl S, Salonen R, et al. Systolic blood pressure during recovery from exercise and the risk of acute myocardial infarction in middle-aged men. Hypertension 2004 Dec;44(6):820-825.

32. Gibbons RJ, Balady GJ, Bricker JT, et al. ACC/AHA 2002 guideline update for exercise testing: summary article. A report of the American College of Cardiology/American Heart Association Task Force on Practice Guidelines (Committee to Update the 1997 Exercise Testing Guidelines). J Am Coll Cardiol 2002 Oct;40(8):1531-1540.

33. American College of Sports Medicine ACSM's Guidelines for Exercise Testing and Prescription. 7th ed. Lippincott Williams \& Wilkins; 2006. 